Received: $\quad 2019.05 .07$ Accepted: 2019.09.03 Published: 2019.12 .12

\section{Sensitivity of Reality Monitoring to Fluency: Evidence from Behavioral Performance and Event-Related Potential (ERP) Old/New Effects}

Authors' Contribution: Study Design A Data Collection B Statistical Analysis C Data Interpretation D Manuscript Preparation E Literature Search F Funds Collection G

Corresponding Authors: Source of support:

Material/Methods:

Results:

Conclusions:

MeSH Keywords:

Full-text PDF:
A 1 Aiqing Nie

B 1 Yueyue Xiao

D 1 Si Liu

F 1 Xiaolei Zhu

E 2 Delin Zhang
1 Department of Psychology and Behavioral Science, Zhejiang University, Hangzhou, Zhejiang, P.R. China

2 Department of Anesthesiology, First Hospital, Zhejiang University, Hangzhou, Zhejiang, P.R. China

Aiqing Nie, e-mail: nieaiq@126.com, Delin Zhang, e-mail: delin-zhang@zju.edu.cn

The Projects of Zhejiang Provincial Natural Science Foundation of China (Grant no. LY17C090003), Humanities and Social Sciences, Ministry of Education, China (Grant no. 17YJA190010), Basic Public Welfare Project of Zhejiang Province, China (Grant No. LGF19H090023), Medical and Health Science and Technology Project of Zhejiang Province, China (Grant no. 2017KY352) supported the current study

Background: Item memory and source memory are differently processed with both behavioral and event-related potential (ERP) evidence. Reality monitoring, a specific type of source memory, which refers to the ability to differentiate external sources from internal sources, has been drawing much attention. Among factors that have an impact on reality monitoring, fluency has not been well-studied. Therefore, the current study aimed to investigate whether fluency could affect reality monitoring, through observations on both behavioral performance and electrophysiological patterns.

Adopting ERP techniques, participants were required either to watch the presentation of a name/picture pair, or to imagine a picture for each displayed name, once (low fluency) or twice (high fluency). Later they completed a reality monitoring task of identifying names as perceived, imagined, or novel items. Behavioral performance was measured, and ERP waveforms were recorded.

Behaviorally, high fluency items were faster and more accurately attributed to the sources than low fluency items. ERP waveforms revealed that late positive component (LPC) occurred for all 4 types of items, while imagined items of low fluency did not record a robust FN400 or late frontal old/new effect.

As results revealed, the factor of fluency does influence reality monitoring in terms of accuracy and responding speed. Meanwhile, for imagined items of low fluency, the absence of FN400 and frontal old/new effect also suggests the sensitivity of reality monitoring to fluency, because these representatives of familiarity-based processing and post-retrieval monitoring are inevitably involved in the process of differentiating internal source from external source.

Environmental Monitoring • Memory • Speech Disorders

https://www.medscimonit.com/abstract/index/idArt/917401

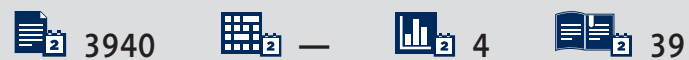




\section{Background}

Within the field of memory research, the dual-process model proposes that memory for events relies on 2 independent processes: familiarity, a relatively automatic and speedy process, and recollection, a more controlled and time-consuming process [1-6]. Episodic memory, which is defined as retrieving information of our daily experienced events, allows us to mentally travel back to our past. Two sub-types of episodic memory have been identified and commonly accepted: Item memory is memory identifying the stimulus as familiar (i.e., distinguishing old items from novel ones); source memory is memory retrieving details within which the event has been experienced. The dual-process model claims that item memory is more familiarity-based versus source memory, while the latter is more recollection-based than the former, which has been supported by many studies $[1,4,7]$. To date, the distinction between these 2 sub-types of episodic memory has been welldocumented, manifesting faster responses for item memory versus source memory, as well as different neural mechanisms.

Consistent with the dual-process model, neural mechanism studies, such as those adopting the event-related potential (ERP) technique to investigate item memory, have described 2 robust patterns paralleling the process of familiarity and recollection, depicting the waveform differences (i.e., old/new effect) between correctly identified studied (or old) items and correctly rejected unstudied (or novel) items. Specifically, an early old/new effect (also known as FN400), which usually peaks at approximately $400 \mathrm{~ms}$ after stimulus presentation over the anterior scalp, with more negative amplitudes for novel versus old items, has been commonly agreed to reflect familiaritybased judgment. In contrast, the late positive component (LPC), another old/new effect happening later, is a component of positive waveforms with more positive amplitudes for old versus novel items that presents at approximately $500 \mathrm{~ms}$ post-stimulus onset over the posterior sites, has been considered to associate with process based on recollection [8-13]. Furthermore, that involvement of FN400 in item memory and source memory is distinct from that of LPC has also been confirmed $[7,14]$. In addition, the waveform differences between these 2 sub-types of memory have also been documented by a third component which merely appears in source memory, the late frontal old/new effect. The component demonstrates that items whose sources are correctly attributed elicit more positive-going waveforms versus new ones over the anterior scalp regions, thus has been considered to correspond with the process of post-retrieval monitoring [11,14-16].

To date, a series of influencing factors have been identified for source memory. Among these, fluency, which is typically defined as a feeling of ease associated with cognitive operation, has received great attention for its influence on a variety of judgments based on conceptual or perceptual similarity [17-19]. For instance, accuracy of source memory was higher for targets presented with the same versus varied contextual details, mainly because of a more natural and fluent processing [18]; besides, N400, a component which reflects semantic processing in source memory, was larger for familiar names in high fluency conditions versus low fluency cases [19].

Considering the multitudinous contextual information being carried in source memory, Johnson et al. proposed a classification for event sources: external sources (i.e., other-generated sources obtained through perceptual processes), and internal sources (i.e., self-generated sources from internal processes, such as reasoning, imagination, or thought) [20]. In view of this, they further declared that source memory contains 3 different manners: to distinguish between different external sources, to distinguish between different internal sources, and to distinguish external sources from internal sources. Most interestingly, as the last manner allows self-generated sources to be distinguished from externally perceived contexts, it is termed "reality monitoring" [21-24].

So far, neural activity studies via the ERP technique have confirmed different patterns on retrieving external sources and internal sources. For instance, from $600 \mathrm{~ms}$ to $1100 \mathrm{~ms}$ poststimulus onset, the ERP waveforms were more positive going over frontal sites when targets were imagined names, whereas the perceived ones did not yield such pattern [21]. However, an ERP study conducted by Rosburg et al. [25] reported that perceived and imagined stimuli elicited similar components of FN400, LPC, and the late frontal old/new effect. These seemingly contradictory results call for further investigation into brain activities during monitoring for internal and external mnemonic details.

In brief, although a large number of source memory studies have been conducted with both behavioral and electrophysiological findings, a comparison of neural mechanisms which underlie distinguishing between stimuli from external and internal sources is needed. Moreover, less is known about how fluency could affect reality monitoring for perceived or imagined items in terms of both behavioral performance and relevant brain activities. In order to address these issues, an ERP experiment was carried out in the current study, in which participants were instructed to retrieve the previous status (perceived or imagined) of the studied items, while the fluency of stimuli was manipulated through prior exposure frequencies (once or twice). It was assumed that significant FN400, LPC, and late frontal old/new effect for both perceived and imagined stimuli could be found. Furthermore, we also expected to see effect of fluency on all these ERP components. 


\section{Material and Methods}

\section{Participants}

A total of 18 young adult university students, who were 19-25 years old and right-handed, participated in this study. Among these students, 10 were females and 8 were males. To estimate the supposed number of participants required in the study, the priori analysis carried out by $G^{*}$ Power 3.1 indicated that the sample size should be 17 , to obtain a small-to-medium sized effect of 0.3 under a standard criteria $(\alpha=0.05,1-\beta=0.80)$ in the 2 (fluency: high versus low) $\times 2$ (form: perceived versus imagined) repeated-measures ANOVAs. Therefore, the number of participants in the current study could meet the requirement.

All participants were native Chinese speakers who claimed that their visual acuity was normal or was corrected-to-normal, neither had they reported history of neurological disorders or mental dysfunctions. Signed informed consent was collected from all participants prior to the experiment, and they received course credits or cash for voluntary participation. The Zhejiang University Research Ethics Board approved the current protocols. All participants were debriefed after the experiment was done.

\section{Design}

The experiment was a reality monitoring task. There were 2 statuses of the studied stimuli (2 levels: perceived and imagined), and these were further manipulated into different levels of fluency (2 levels: low and high). To specify, the levels of fluency were defined and determined by presenting times of stimuli during encoding, i.e., stimuli presented once were of low fluency and twice were of high fluency.

\section{Materials}

The stimuli were 180 pictures and 230 words. Pictures were black and white meaningful line pictures of namable objects from Snodgrass and Vanderwart [26], which were revised to Chinese version in a previous study [27]. Words were all 2-character Chinese words, 180 of which were names of the objects shown in the pictures, while the remaining 50 were names of other objects. The formal experiment comprised 160 pictures and 200 names, while others were reserved for practical trials. To reduce participant fatigue and to avoid interferences across different stimuli, the whole experiment included 4 distinct blocks, each had 40 pictures and 50 names. Across these blocks, the pictures were equivalent in the scores of naming difficulty, familiarity, perceptual complexity, as well as imaging consistency, as those provided by Snodgrass and Vanderwart [26], and names were also balanced in terms of frequency, stroke, spelling, and pronunciation, as described by
Nie et al. [27]. In each study block, there were 20 pictures and 40 names. There were 50 names presented in each test block: 10 perceived with low fluency (PWLF), 10 perceived with high fluency (PWHF), 10 imagined with low fluency (IWLF), 10 imagined with high fluency (IWHF), and 10 new names.

\section{Procedure}

Practical trials with appropriate feedbacks were provided for participants to ensure that they had fully understood the task before entering to the formal experiment. Except for the feedback portion and the stimuli used, all protocols were the same between the practice and formal experiment. Task instructions were given to each participant before each study and test block separately. During the study phase of each block, pairs of a name and its corresponding picture were randomly shown in the perceived condition, with the names always being displayed above the pictures; while in the imagined condition, pairs of a name and a white blank screen were presented. For the name/picture pairs, participants were instructed to decide whether the simultaneously presented name and picture matched or not, by pressing "F" or "J" keys on the keyboard, respectively. In fact, all pairs were matched, and the purpose of the task was to ensure that participants actually focused on all stimuli (i.e., both name and picture). For the name/screen pairs, participants were informed to imagine a picture for each displayed name, and they were instructed to press the "F" key on the keyboard when a relevant picture had been imagined, if not, press the "J" key. The next pair was presented regardless of whether key pressing was made or not. The 2 types of pairs were hereafter referred to as perceived and imagined pairs, respectively. One half of the perceived and imagined pairs was shown once, while the other half was presented twice. Thus, PWLF, PWHF, IWLF, and IWHF were equivalent in amount. Each pair was displayed for $2000 \mathrm{~ms}$, and the interstimulus interval (ISI) was $1500 \pm 200 \mathrm{~ms}$.

During the memory test, names were randomly displayed in the monitor center, each was presented an interval of $1500 \mathrm{~ms}$ with an ISI of $1500 \pm 200 \mathrm{~ms}$. The test was set using the 3-key paradigm, a widely used paradigm for investigating source memory $[14,27,28]$, in which participants were instructed to press 3 different keys on the keyboard to attribute names of objects to previously perceived items, previously imagined ones, and new ones. After an entire block of study and test phases, a 5-minute interval for rest was sandwiched before the next block, in order to prevent interferences across blocks and to reduce participant fatigue.

Stimuli in both study and test phases were programmed by E-prime software v3.0 (Psychology Software Tools, Inc.), and were presented in a black background. The screen had a refresh frequency of $75 \mathrm{~Hz}$ and a resolution of $1024 \times 768$ pixels. 
The vertical and horizontal angles of the perceived pictures or white blank screens were $5.34^{\circ}$, and those for the names were $5.34 \times 2.67^{\circ}$. All names were shown in white bold font. No stimuli were re-presented across blocks. During the whole experiment, the order of the blocks was pseudo-randomized. Participants were instructed to press the keys applying their thumb and index fingers, and for each participant, the key pressings were balanced among different blocks. Meanwhile, participants were required to fixate the eyes on the screen center and to minimize their eye blinks during the presentation of stimuli. Participants were also encouraged to make responses quickly and accurately at the same time. Flow chart of the experimental procedure is illustrated in Figure 1.

\section{Electrophysiological recording}

The whole experiment was done in an electrically shielded booth. The EEG (electroencephalogram) was constantly recorded from pre-defined $32 \mathrm{Ag} / \mathrm{AgCl}$ electrodes with Synamp amplifiers, stretching from the $10 / 20$ system. The external canthi of both eyes placed electrodes were used to record the horizontal EOG (electro-oculogram), while the recording of vertical EOG was collected from the electrode on the supra-orbital ridge of the left eye as well as the one on its infra-orbital ridge. For online reference, the right mastoid was chosen, whereas in the offline circumstance, EEG recordings were re-referenced algebraically to the mean value of both mastoids. We amplified the signal with a gain of 250 , sampling at $500 \mathrm{~Hz}$, and a band-pass filter of $0.05-70.00 \mathrm{~Hz}$ was adopted. Besides, we also kept all electrode impedance not exceeded $5 \mathrm{k} \Omega$.

\section{Results}

\section{Behavioral analyses and the data}

Accuracy of key pressing during the study phase was always above $95 \%$ for all participants. Hence, only the testing performance was concerned. Data were analyzed applying IBM SPSS Statistics V22, and those less or greater than 3 standard deviations were excluded. All inferential analyses were conducted at the alpha level of 0.05. A 2-way repeated-measures ANOVA was conducted on accuracy and reaction time, with status (2 levels: perceived and imagined) and fluency (2 levels: low and high) as variables.

With regard to accuracy, the main effects of both factors were significant, $F(1,17)=38.673, P<0.001, \eta^{2}=0.659$ and $F(1,17)=163.718, P<0.001, \eta^{2}=0.891$, respectively, indicating that the accuracy was much higher for names of high fluency than those of low fluency, and the performance of perceived trials was more accurate than imagined ones. For reaction time, the ANOVA also revealed reliable main effects for both status and fluency, $F(1,17)=120.003, P<0.001, \eta^{2}=0.857$ and $F(1,17)=30.401, P<0.001, \eta^{2}=0.603$, as well as their interaction, $F(1,17)=7.769, P<0.05, \eta^{2}=0.280$. Simple effect analyses confirmed much longer responses in low fluency versus high fluency cases for both perceived and imagined names, $F(1,17)=134.46, P<0.001$ and $F(1,17)=69.37, P<0.001$, and response time was shorter for perceived names vs. imagined ones, $P<0.001$. Furthermore, responses to new names were longer than old ones, $F(1,17)=52.225, P<0.001$.

\section{ERP analyses and the data}

Before averaging data, similar to previous studies [6,29], a semi-automatic procedure was done to correct eye artifact. Those eye blinks or other movements that caused a fluctuation exceeding $\pm 100 \mu \mathrm{V}$ would contaminate the data and were thus excluded based on PCA algorithm before collapsing [30]. An extraction of epochs from $200 \mathrm{~ms}$ pre-stimulus onset to 1000 ms post-stimulus onset was elicited from the consecutive recording, and the waveforms were corrected with the prestimulus latency. The waveforms were separately collapsed for different correctly identified trials of 4 types of names (PWLF, PWHF, IWLF, and IWHF) and the new trials.

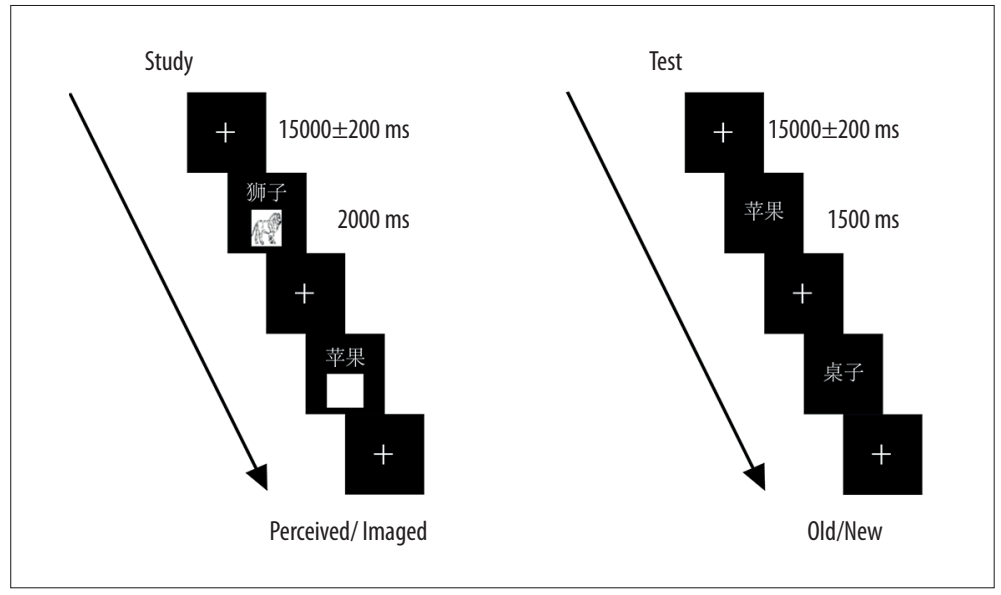

Figure 1. Flow chart of the experimental procedure and some stimulus exemplars. 


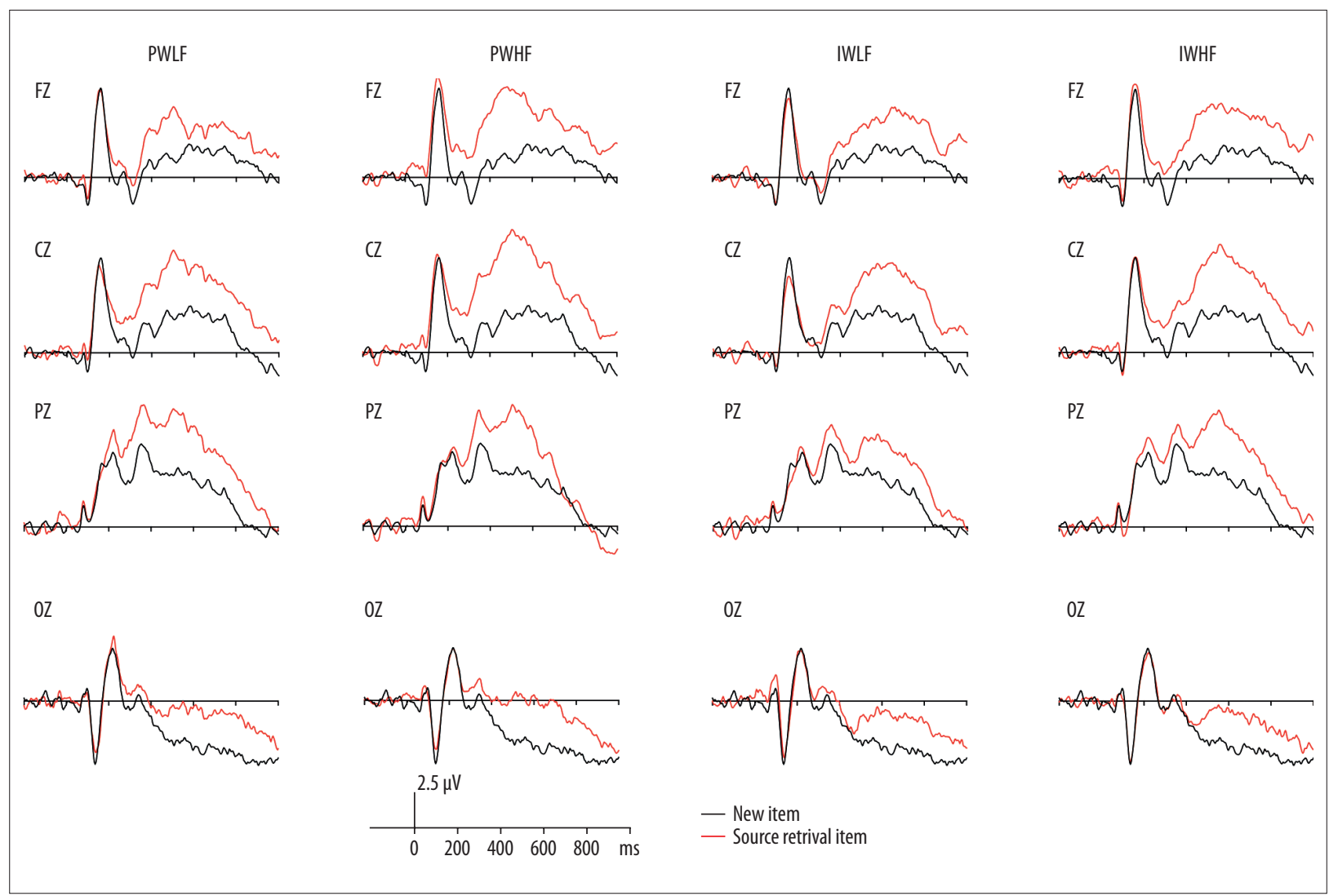

Figure 2. Grand average waveforms depicted at selected electrodes for source correctly retrieved names and new ones, respectively. PWLF - perceived with low fluency; PWHF - perceived with high fluency; IWLF - imagined with low fluency; IWHF - imagined with high fluency.

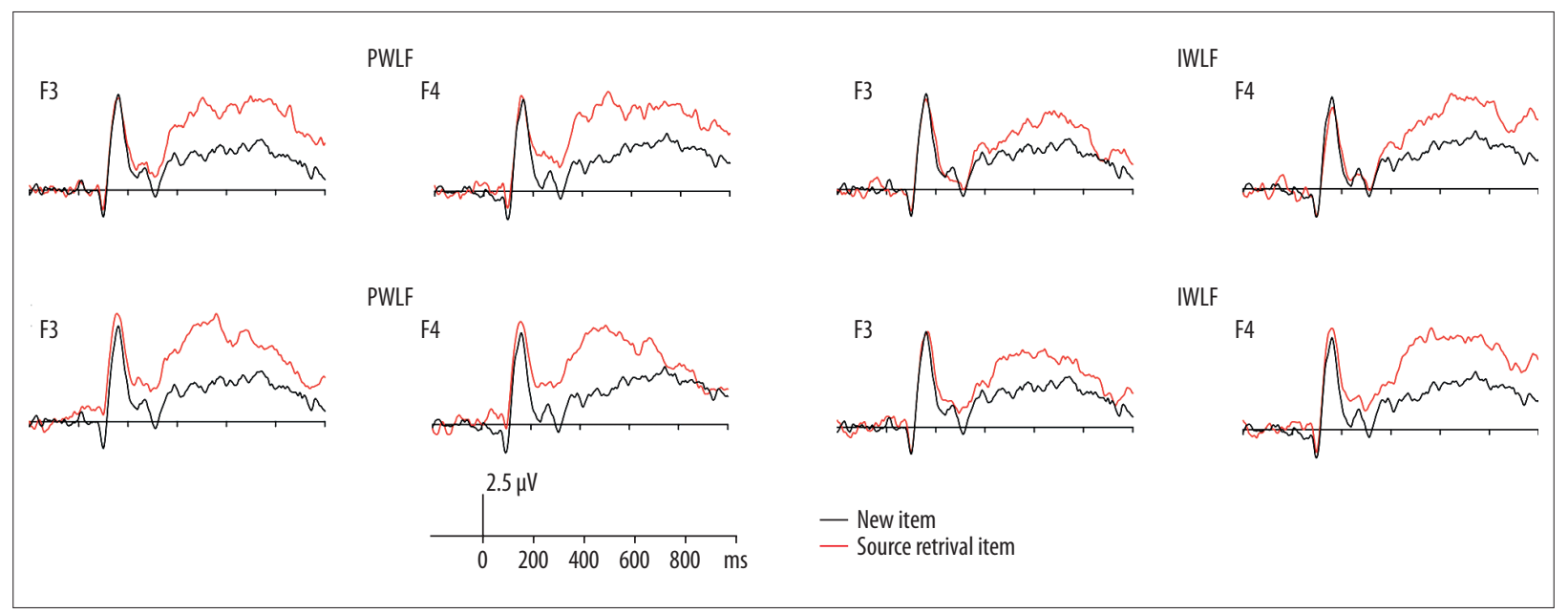

Figure 3. Grand average waveforms over bilateral frontal electrodes separately for source correctly retrieved items and new ones, respectively. The condition descriptions were the same as in Figure 2.

The grand average waveforms for 4 types of names (PWLF, PWHF, IWLF, and IWHF) and new names were presented in Figure 2. Figure 3 presents the diagram of the waveforms over bilateral frontal electrodes. Figure 4 depicts the topographical maps obtained by subtracting the waveforms of the names with correctly retrieved sources from those of novel names. The amplitudes of the source correctly retrieved PWLF, PWHF, IWLF, and IWHF names were separately compared with those of new ones. The latency window was 350-500 and 500-650 ms for FN400 and LPC, respectively. The repeated-measures ANOVA 


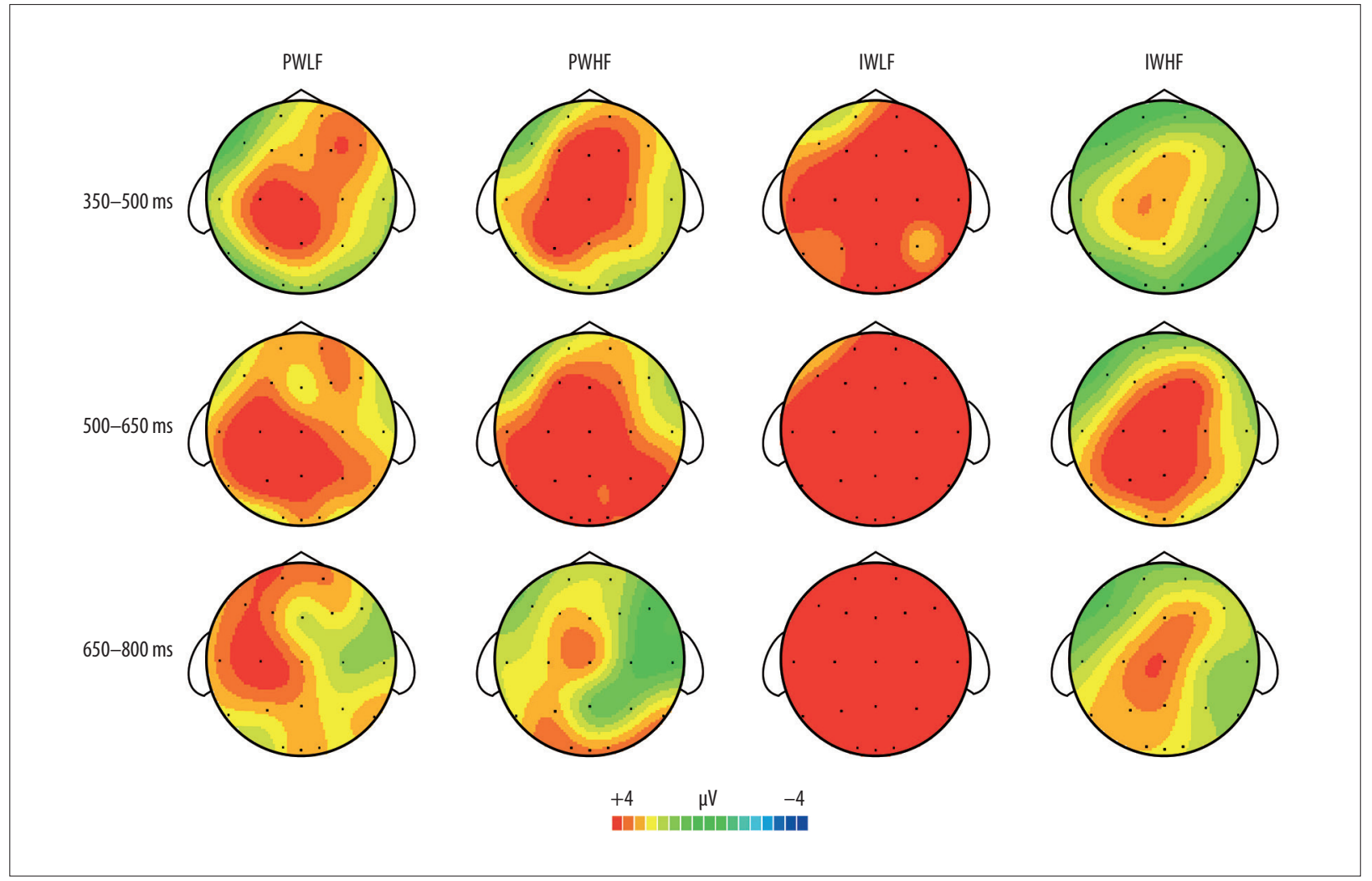

Figure 4. Scalp topographies of FN400, LPC, and the late frontal old/new effect. FN400, also known as the early old/new effect; LPC - late positive component.

of 2 (item type: source correctly retrieved and new) $\times 4$ (cortical region: frontal [FZ], central [CZ], parietal [PZ], and occipital [OZ]) was separately performed for both components. The late frontal old/new effect was calculated in the time latency of 650-800 ms, with the variables of cortical region (2 levels: F3 and F4) by item type (2 levels: source correctly retrieved and new). Merely data relevant to the item type were given concern. Greenhouse-Geisser correction was used, when necessary.

During 350-500 ms, considering PWLF, the item type held a robust main effect, $F(1,17)=21.333, P<0.001, \eta^{2}=0.557$, and an interaction between the two variables, $F(3,51)=4.852, P<0.05$, $\eta^{2}=0.222$. Simple effect analyses confirmed that the waveforms of the source correctly retrieved PWLF trials were larger than those of new trials, and this difference was shown over the frontal, central, parietal, and occipital sites, $F(1,17)=13.406, P<0.01$, $\eta^{2}=0.441 ; F(1,17)=21.175, P<0.001, \eta^{2}=0.555 ; F(1,17)=19.156$, $P<0.001, \eta^{2}=0.530 ;$ and $F(1,17)=11.558, P<0.01, \eta^{2}=0.405$. It also elicited a reliable main effect of item type for PWHF, and a significant interaction of item type by cortical region, $F(1,17)=27.385, P<0.001, \eta^{2}=0.617$ and $F(3,51)=4.769, P<0.05$, $\eta^{2}=0.219$. Similarly, further analyses suggested that PWHF words whose source were accurately attributed elicited waveforms of a more positive trend than new names over all the 4 concerned regions, $F(1,17)=11.966, P<0.01, \eta^{2}=0.413$;
$F(1,17)=26.474, P<0.001, \eta^{2}=0.609 ; F(1,17)=24.086, P<0.001$, $\eta^{2}=0.586$; and $F(1,17)=21.613, P<0.001, \eta^{2}=0.560$. No significant effect of item type appeared for IWLF, $P S>0.1$, but its main effect showed in IWHF items, $F(1,17)=3.685, P<0.05, \eta^{2}=0.178$. Thus, the findings above indicated a robust FN400 for PWLF, PWHF, and IWHF.

During the 500-650 ms, item type yielded a main effect which occurred in PWLF, PWHF, IWLF, and IWHF trials, $F(1,17)=18.832$, $P<0.001, \eta^{2}=0.526 ; \quad F(1,17)=47.466, P<0.001, \eta^{2}=0.736$; $F(1,17)=20.861, P<0.001, \eta^{2}=0.551$; and $F(1,17)=8.710, P<0.01$, $\eta^{2}=0.339$, which strongly proved a robust LPC for all 4 types of names.

For the latency window of 650-800 ms, item type exhibited a reliable main effect in PWLF, $F(1,17)=9.283, P<0.01, \eta^{2}=0.353$. Considering PWHF, there was not only an effect of item type, $F(1,17)=5.901, P<0.05, \eta^{2}=0.258$, but also a 2 -way interaction which only reached marginal significance, $F(1,17)=3.879$, $P<0.1, \eta^{2}=0.186$. Simple effect analyses confirmed more positive-going waveforms for the source correctly retrieved PWHF trials, when compared with new items over $\mathrm{F} 3, F(1,17)=7.871$, $P<0.05, \eta^{2}=0.316$. As for imagined items, no effects were found for IWLF, $P S>0.1$. However, main effect of item type was presented for IWHF, $F(1,17)=4.797, P<0.05, \eta^{2}=0.220$, as well as 
the interaction, $F(1,17)=6.156, P<0.05, \eta^{2}=0.266$, with more positive-going waveforms over $\mathrm{F} 4$ for source correctly retrieved IWHF trials than new ones, $F(1,17)=8.016, P<0.05, \eta^{2}=0.320$, based on results of simple effect analyses. Thus, PWLF, PWHF, and IWHF all confirmed the reliable late frontal old/new effect.

\section{Discussion}

The current experiment was carried out to determine to what extent the factor of fluency could affect the activity of reality monitoring, from a dual behavioral-electrophysiological perspective. In particular, adopting electrophysiological technique, the objective of the current study was to probe whether stimulus fluency would have an affection on ERP old/new effects, and whether could reveal a different pattern between perceived and imaged items. A general discussion concerning our findings would be given in the following parts.

Regarding the behavioral perspective, previous studies have suggested a higher accuracy for events with external versus internal sources [21,31-33], similarly to that perceived names were more accurate than imagined names in the current study. Additionally, in line with findings in previous studies [21,25,31-33], we also found that less time was spent on responding to perceived items versus imagined items. Besides, for both perceived and imagined items, shorter response and greater accuracy were found with high fluency items, instead of low fluency items, implicating that fluency does have an impact on reality monitoring.

Turning to the brain activities, the current source correctly identified PWLF, PWHF, and IWHF names elicited a robust FN400, and all 4 types of names yielded the robust LPC, while the late frontal old/new effect for PWLF, PWHF, and IWHF names was significant. Quite evidently, these findings not only offered strong evidence for old/new effects, but also proved an effective influence of fluency on the patterns of these effects. Such deduction was due to the fact that there was no the FN400 and late frontal old/new effect in the case of the imagined items of low frequency. As mentioned before, reliable FN400 and LPC were found, suggesting that reality monitoring relied on both familiarity- and recollection-based processes, which were nicely in line with the findings of previous study [14]. Furthermore, the late frontal old/new effect occurred more evidently in source correctly retrieved PWLF, PWHF, and IWHF cases. These findings suggested the process of successful postretrieval monitoring that has been proved and documented in many studies [11,14-16].

Speaking of processing in the frontal site, to date, a large number of studies have widely verified the engagement of the frontal region in the retrieval of a range of contextual details, with converging evidence from studies of normal participants, aging populations, and patients as participants [34-37]. Thus, a reliable late frontal old/new effect found in the current study was correspond to previous studies. Furthermore, we did not only reveal the late frontal old/new effect in retrieving perceived sources, but also in retrieving imagined sources, which suggested that the frontal region is involved in retrieving both external and internal sources.

Apart from replicating the standard late frontal old/new effect, brain activities dissociation between internal and external sources were also identified, which was correspond to earlier findings $[21,25,32,33]$. Furthermore, focus was given on the impact of fluency on reality monitoring, as a mediation of fluency upon which has not been considered. Hopefully, we identified the dissociation of the sensitivity between internal and external sources to fluency, particularly for the imaged cases, where both the components of FN400 and late frontal old/new effect were more significant for the source correctly identified IWHF, when compared with IWLF, which demonstrated that the fluency-based process affected the remembering of imaged items.

We also tried to comprehend the behavioral results and ERPS waveforms from a perspective of microgenetic theory, as the cognitive process explained by this theory can also be applied to a number of different fields of interest [38]. Microgenetic theory posits that memory for a perceived event develops at the same time as its perception, remaining silent until it is activated, thus recall accuracy of an event is associated with the extent to which the content of the episode in the initial perception phase could be revived [39]. Consistent with this assumption, our results showed that in the perceived condition, high fluency words were faster and more accurately recalled than low fluency words, as encountering high fluency words was an event of repeated exposure, which could be more easily retrieved. For electrophysiological result of perceived stimuli, a pattern showing 3 recurrent stages of old/new effect (i.e., FN400, LPC, and the late frontal old/new effect), these findings were analogous as the recurrence of information processing in perception illustrated in Pąchalska et al. study [39], supports the basic premise of microgenetic theory which is rooted in process thought $[38,39]$. As for those words whose sources were incorrectly retrieved, though we did not explicitly state in the result section, that less accurate source memory was for low frequently processed stimuli than words of high frequency, according to microgenetic theory which is also formed based on findings of observable symptoms of brain damage, could be accounted for differences in processing in the ventral and dorsal streams during perception [38]. As we did not focus on the perception processing, it would be a logical next step for later research to explore how the processing in left/right ventral and dorsal streams which is at temporal and parietal 
sites respectively, influence the accuracy, reaction times, and waveforms of retrieval.

\section{Limitations and further directions}

In the current study, several limitations should be mentioned. First, merely differences between perceived and imaged events were compared, while whether similar patterns could be confirmed for other modalities of self-generated or other-generated sources, such as seen, spoken, and thought ones, remains unclear. Second, we should mention that the event, stored in our episodic memory, could be occasionally happened or repeatedly happened, could be perceived with or without personal feelings, and could be processed with or without the help of semantic knowledge. Thus, when fluency was manipulated through a perceptual way in our study in which words has been presented once or twice, possibly causing the events more "episodic" or "semantic", according to microgenetic theory [39]. Therefore, it is necessary for future research to rule out the possibilities that the difference is not brought by perceptual fluency but by types of events; at the same time, as mentioned before, fluency includes different styles [17-19],

\section{References:}

1. Cooper E, Greve A, Henson RN: Assumptions behind scoring source versus item memory: Effects of age, hippocampal lesions and mild memory problems. Cortex, 2017; 91: 297-315.

2. King DR, de Chastelaine M, Elward RL et al: Dissociation between the neural correlates of recollection and familiarity in the striatum and hippocampus: Across-study convergence. Behav Brain Res, 2018; 354: 1-7

3. Wang WC, Brashier NM, Wing EA et al: Knowledge supports memory retrieval through familiarity, not recollection. Neuropsychologia, 2018; 113: 14-21

4. Wynn SC, Daselaar SM, Kessels RP, Schutter DJ: The electrophysiology of subjectively perceived memory confidence in relation to recollection and familiarity. Brain Cogn, 2019; 130: 20-27

5. Malejka S, Bröder A: No source memory for unrecognized items when implicit feedback is avoided. Mem Cogn, 2016; 44(1): 63-72

6. Nie A, Griffin M, Keinath A et al: ERP profiles for face and word recognition are based on their status in semantic memory not their stimulus category. Brain Res, 2014; 1557: 66-73

7. Ventura-Bort C, Dolcos F, Wendt J et al: Item and source memory for emotional associates is mediated by different retrieval processes. Neuropsychologia, 2017 [Epub ahead of print].

8. Leynes PA, Batterman A, Abrimian A: Expectations alter recognition and event-related potentials (ERPs). Brain Cogn, 2019; 135: 103573

9. Küper K, Zimmer HD: The impact of perceptual changes to studied items on ERP correlates of familiarity and recollection is subject to hemispheric asymmetries. Brain Cogn, 2018; 122: 17-25

10. Kueper K: On the functional significance of retrieval mode: task switching disrupts the recollection of conceptual stimulus information from episodic memory. Brain Res, 2018; 1678: 1-11

11. Leynes PA, Crawford CJ: Event-related potential (ERP) evidence that encoding focus alters recollected features. Brain Cogn, 2018; 127: 42-50

12. Bader R, Mecklinger A: Separating event-related potential effects for conceptual fluency and episodic familiarity. J Cogn Neurosci, 2017; 29(8): 1402-14

13. Herweg NA, Sommer T, Bunzeck N: Retrieval demands adaptively change striatal old/new signals and boost subsequent long-term memory. J Neurosci, 2018; 38(3): 745-54

14. Leynes PA, Askin B, Landau JD: Visual perspective during remembering: ERP evidence of familiarity-based source monitoring. Cortex, 2017; 91: 157-68 so the replaceable conceptual fluency should be considered in further investigations to explore the sensibility of reality monitoring to fluency. Lastly, a caveat would be that the current fluency was controlled during the study phase, while the fluency triggered by the tests also needs further consideration.

\section{Conclusions}

In terms of sensitivity of reality monitoring to fluency, the current data provides evidences of both behavioral differences and ERP old/new effects. For both perceived and imagined items, the responses were much slower for items of low fluency versus those of high fluency, and accuracies were higher for high fluency trials. As for ERP old/new effects, both FN400 and late old/new effect were absent in IWLF items, revealing impact of fluency on the familiarity-based process and postretrieval monitoring for imagined items.

\section{Conflicts of interest}

None.

15. Mao X, Wang Y, Wu Y, Guo C: Self-referential information alleviates retrieval inhibition of directed forgetting effects - an ERP evidence of source memory. Front Behav Neurosci, 2017; 11: 187

16. Zheng Z, Li J, Xiao F et al: Unitization improves source memory in old er adults: an event-related potential study. Neuropsychologia, 2016; 89: 232-44

17. Wang W, Li B, Gao C, Guo C: The temporal dynamics of perceptual and conceptual fluency on recognition memory. Brain Cogn, 2018; 127: 1-12

18. Komes J, Schweinberger SR, Wiese H: Fluency affects source memory for familiar names in younger and older adults: Evidence from event-related brain potentials. Neuroimage, 2014; 92: 90-105

19. Kurilla BP: Enhanced processing fluency leads to biases in source memory. Q J Exp Psychol, 2011; 64(8): 1609-31

20. Johnson MK, Hashtroudi S, Lindsay DS: Source monitoring. Psychol Bull, 1993; 114(1): 3-28

21. Garrison JR, Bond R, Gibbard E et al: Monitoring what is real: The effects of modality and action on accuracy and type of reality monitoring error. Cortex, 2017; 87: 108-17

22. Le Bigot L, Knutsen D, Gil S: I remember emotional content better, but I'm struggling to remember who said it! Cognition, 2018; 180: 52-58

23. Simons JS, Garrison JR, Johnson MK: Brain mechanisms of reality monitoring. Trends Cogn Sci, 2017; 21(6): 462-73

24. Mac Giolla E, Ask K, Granhag PA, Karlsson A: Can reality monitoring criteria distinguish between true and false intentions? J Appl Res Mem Cogn, 2019; 8(1): 92-97

25. Rosburg T, Mecklinger A, Johansson M: Strategic retrieval in a reality monitoring task. Neuropsychologia, 2011; 49(10): 2957-69

26. Snodgrass JG, Vanderwart M: A standardized set of 260 pictures: Norms for name agreement, image agreement, familiarity, and visual complexity. J Exp Psychol Learn Mem Cogn, 1980; 6(2): 174-215

27. Nie A, Guo C, Shen M: The influence of the testing paradigm on location source retrieval: An event-related potentials study. Acta Psychol Sin, 2011; 43(05): 473-82

28. Evans LH, Herron JE: Pre-retrieval event-related potentials predict source memory during task switching. Neuroimage, 2019; 194: 174-81 
29. Nie A, Li M, Ye J: Lag-length effect on repetition priming of famous and unfamiliar faces: Evidence from N250r and N400. Neuroreport, 2016; 27(10): 755-63

30. Nowagk R, Pfeifer E: Unix implementation of the ERP evaluation package (EEP 3.0). Annu Rep MPI Cogn Neurosci, 1996; 124

31. Subramaniam K, Gill J, Slattery P et al: Neural mechanisms of positive mood induced modulation of reality monitoring. Front Hum Neurosci, 2016; 10 581

32. Kensinger EA, Schacter DL: Neural processes underlying memory attribution on a reality-monitoring task. Cereb Cortex, 2005; 16(8): 1126-33

33. Turner MS, Simons JS, Gilbert SJ et al: Distinct roles for lateral and medial rostral prefrontal cortex in source monitoring of perceived and imagined events. Neuropsychologia, 2008; 46(5): 1442-53
34. Meusel LA, Grady CL, Ebert PE, Anderson ND: Brain-behavior relationships in source memory: Effects of age and memory ability. Cortex, 2017; 91 : 221-33

35. Monge ZA, Stanley ML, Geib BR et al: Functional networks underlying item and source memory: Shared and distinct network components and agerelated differences. Neurobiol Aging, 2018; 69: 140-50

36. Garrison JR, Fernandez-Egea E, Zaman R et al: Reality monitoring impairment in schizophrenia reflects specific prefrontal cortex dysfunction. Neuroimage Clin, 2017; 14: 260-68

37. Powell PS, Strunk J, James T et al: Decoding selective attention to context memory: An aging study. Neurolmage, 2018; 181: 95-107

38. Pachalska M, MacQueen BD, Cielebak K: The creative potential of microgenetic theory. Acta Neuropsychol, 2018; 16(2): 125-55

39. Pąchalska M, Góral-Pólrola J, Mueller A, Kropotov JD: Neuropsychology and the neurophysiology of perceptual microgenesis. Acta Neuropsychol, 2017; 15(4): 365-89 